\title{
Experimentelle Studien über die Neotenie bei Amphibien.
}

\author{
Von \\ Toratoshi Taniguchi. \\ Ius dem Anatomischen Institut der Keio Universität, Tokyo.
}

Mit 3 Textfiguren.

Bezüglich der Faktoren, durch welche die Neotenje hervorgerufen wird, sind seit Duméril ('67) von vielen Autoren folgende verschiedene Meinungen geäussert worden : Die übermässige oder mangelhafte Luftmenge in Wasser (von Chauvin), die Vererbung der Prädisposition für Neotenie (Fremery), die Verletzung der Extremitäten oder Schwänze (Duméril, Kammerer), überschüssige (Gadow, Sasaki) oder ungenügende (Woltersdorff) Ernährung, die niedrige Temperatur (Zeller, Barfurth, Hamann, Sasaki, Boettger) und die geographische Lage des Tümpels, worin die Tiere wohnen.

Die zahlreichen Fütterungsversuche an Amphibien führen erst heute zu dem Schluss, dass die Entstehung der Neotenie mit der Funktion der innersekretorischen Organe (Schilddrüse, Thymus und Hypophyse) in enger Beziehung steht, besonders mit der Schilddrüse. Neuerdings ist aber noch von anderen Autoren (Cramer, Mills, Hart, Adler, Nishimura u. a.) mitgeteilt worden, dass die niedrige Temperatur auf die histologischen Befunde der Schilddrüse eine auffällige Veränderung ausüben kann. Noch im Jahre 1929 hat Huxley mitgeteilt, dass die Schilddrüse der Kaulquappen als primitiver Temperaturregulator dient.

Eine eingehendere Untersuchung über die Beziehungen zwischen der Entstehung der Neotenie und der niedrigen Temperatur sowie der immersekretorischen Organe ist heute noch sehr erwünscht. Diesbezüglich etwas beizutragen, habe ich die vorliegende Untersuchung an Larren 
von Hynobius fuscus und an Kaulquappen von Bufo vulgaris sowie Rana japonica angestellt.

Versuch I. Über die Wirkung der Schilddrüse und ihres Präparates auf Larven von Hynobius fuscus und Kaulquappen.

Als Material wurden die Kaulquappen von Rana japonica von ca. $20 \mathrm{~mm}$ Körperlänge und die frisch ausgebrüteten Larven von Hynobius fuscus verwendet. Für Behälter benutzte ich kleine Glaskufen. Die beiden Versuchstiere wurden in 3 Gruppen geteilt; die der ersten Gruppe wurden mit Thyreoideum siccum von Sankyo Co. Tokyo, die der zweiten mit Schilddrüsen-Pulver des Rindes, das so hergestellt wurde, dass die frische Schilddrüse in dümne Stücke geschnitten, vollständig getrocknet und im Mörser zerrieben wurde, und die der dritten als Kontroll mit natürlicher Nahrung gefüttert. In jede Gruppe kamen 20 Larven. Das Experiment begann am 3. Mai 1925 und wurde am 13. Juni abgeschlossen.

Durch diesen Versuch konnte ich konstatieren, dass die Wirkung (Beschleunigung der Entwicklung und der Metamorphose) des Thyreoideum viel stärker als die des Schilddrüsen-Pulvers des Rindes und bei Kaulquappen stärker als bei Hynobius war. Bei den Kaulquappen, die mit Thyreoideum gefüttert wurden, ist die linke vordere Extremität schon am zweiten Versuchstage durchgebrochen. Aus diesen Befunden fragt es sich, warum die Differenz der Wirkung je nach den Tierarten entstanden ist. Ich bin der Meinung, dass die Metamorphose im allgemeinen bei Anuren in einem höheren Grad als bei Urodelen vor sich geht. Auch mag die Schilddrüse bei jenen immer, bei diesen nur gerade vor oder während der Metamorphose funktionieren, wie Uhlenhuth es schon bemerkte. Daraus könnte leicht erklärt werden, dass die neotenischen Larven bei Urodelen viel häufiger als bei Anuren angetroffen werden könmen.

Versuch II. Über die Wirkung des Thymus-Präparates auf Metamorphose von Kaulquappen.

Es handelt sich um die Kaulquappen von Bufo vulgaris japonicus, die beim Beginn des Versuches durchschnittlich $30 \mathrm{~mm}$ Körperlänge besassen. Die gebrauchten Behälter und sonstige Manipulationen sind ganz gleich wie beim Versuch I. Der Versuch wurde am 7. Nai 1928 begonnen und am 13. Mai abgeschlossen. Die Versuchstiere wurden in 4 Gruppen geteilt und in jede Gruppe kamen 20 Kaulquappen. Die Tiere der ersten Gruppe wurden mit Thymus-Pulver von Sankyo Co. 
Tokyo, die der zweiten mit demselben ausserdem noch mit gleicher Menge von Thyreoideum siccum ron derselben Co., die der dritten nur mit Thyreoideum und die der letzteren als Kontroll mit gemischter Nahrung gefüttert. Die abgegebene Thyreoideummenge bei dritter Gruppe war aber doppelt so gross als bei der zweiten.

Bezüglich der Metamorphose besteht es kein nemmenswerter Unterschied zwischen den Tieren der ersten Gruppe (Thymustier) und Kontrolltieren. I)ie Tiere der zweiten Gruppe (Thyreoideum-ThymusTier) und der dritten (Thyreoideumtier) erfuhren eine starke Beschleunigung der Metamorphose und die letzteren viel stärker als die ersteren. Aus diesen Befunden geht klar hervor, dass das Thymus-Präparat auf Kaulquappen gar nicht oder nur ein wenig einwirkt. Die Wirkung des Thymus-Präparates ist schon von vielen Forschern oft umstritten worden. Diese Frage endgültig zu entscheiden, bedarf es noch weiterer Untersuchungen.

Versuch III. Über die Beziehungen zwischen Neotenie und niedriger Temperatur.

1. Über die Wirkung der Kälte auf Entwicklung und Irachstum von Amphibienlarven.

a. Versuch an Bufo vulgaris japonicus.

Als Versuchstiere wurden die Kaulquappen von Bufo vulgaris japonicus gebraucht, die in einem Tümpel der nächsten Umgebung von Tokyo-Stadt ausgebrütet und dort ganz frei lebten. Beim Beginn des Versuches hatten die Tiere gut entwickelte hintere Extremitäteu, aber noch keine vorderen. Sie wurden in zwei Gruppen eingeteilt, die je 24 Larven enthielten. Die Gruppe A (Kontroll) wurde bei Zimmertemperatur, Gruppe B (Kältetier) in einem Kühlkasten ron $10^{\circ} \mathrm{C}$ gehalten und als Nahrung wurden den beiden gekochter Reis, Fischfleisch, Wasserpflanzen, Leberpulver des Rindes u. a. gegeben.

Die Kontrolltiere wandelten sich alle in normaler Weise und Zeit um. Die Metamorphose erfolgte hier schon am 2. Versuchstage und war am 20. vollendet. Dagegen war die Umwandlungszeit bei Kältetieren bedeutend verlängert. Ich fand erst am 20. Tersuchstage ein ungewandeltes Tier, dem schon seit dem 9. Tersuchstage die rechte vordere Lxtremität ausgebrochen ist. Der Verlauf des Experimentes ist in folgenter Tabelle übersichtlich angegeben. 
Tabelle 1 .

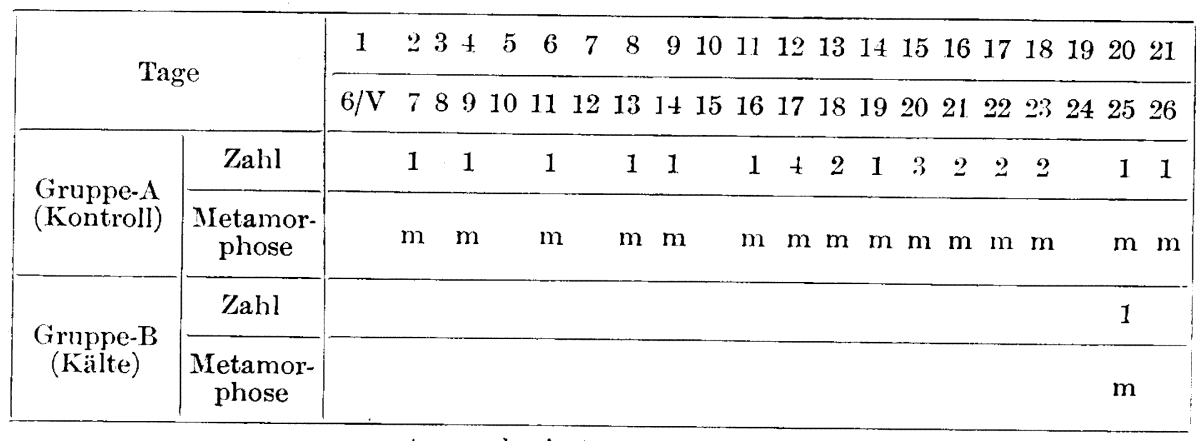

$\mathrm{m}=$ metamorphosiert.

Die nicht metamorphosierten Kältetiere teilte ich am 30. Mai (25. Versuchstage) in zwei Gruppen ein. Eine Gruppe (Gruppe C) wurde bei Zimmertemperatur untergebracht, sie wandelten sich fast alle binnen 10 Tagen um. Die anderen Tiere (Gruppe D) wurden dauernd in Kälte gehalten; die 4 Tiere dieser Gruppe haben metamorphosiert und alle sonstigen starben binnen 52 Versuchstagen. Der Verlauf dieser zwei Gruppen (C und D) ist in folgender Tabelle angegeben.

Tabelle 2 .

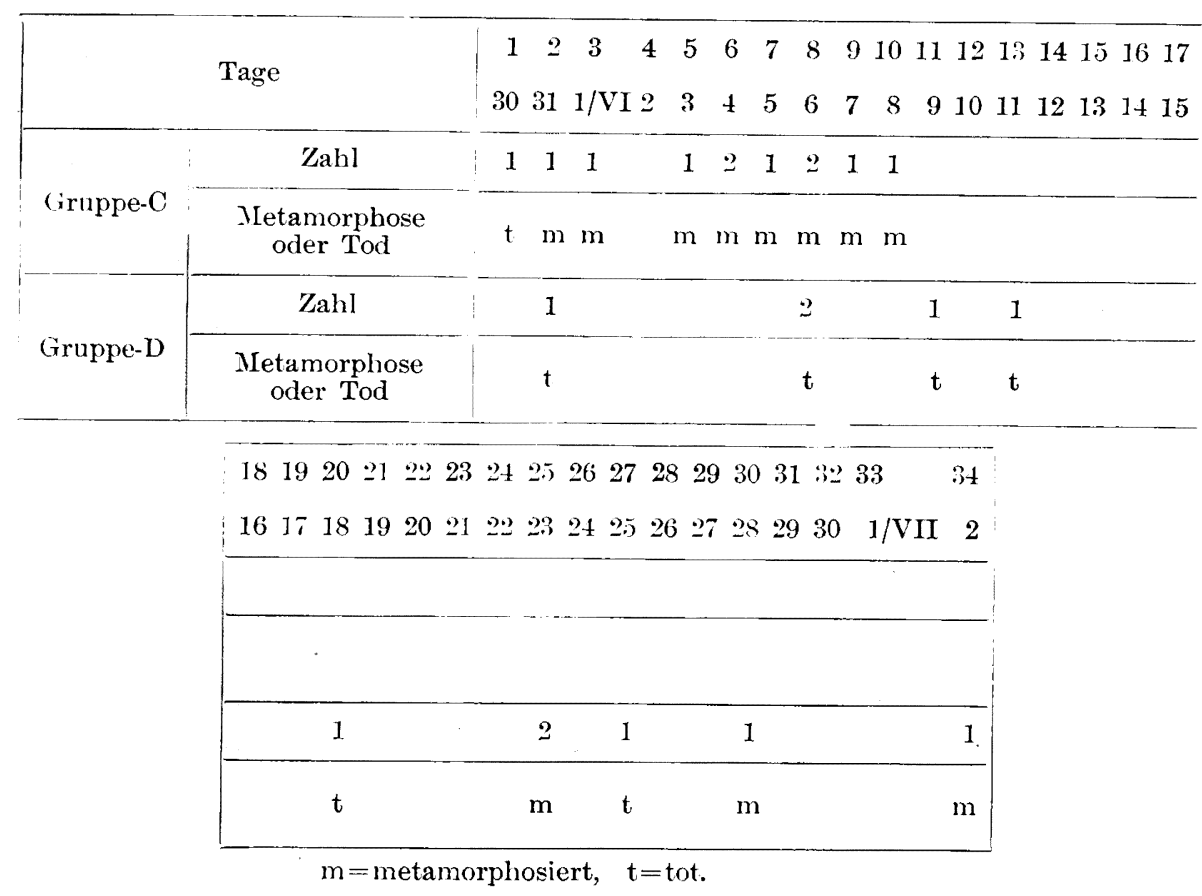


Die mmgewandelten oder gestorbenen Tiere wurden alle in 10\% Formol fixiert und Körpergewicht und -länge gemessen. Die durchrehnittlichen Masse sind in folgender Tabelle angegeben.

Tabelle 3 .

\begin{tabular}{|c|c|c|c|}
\hline Arten & Körpergewicht & Stammlänge & Gesamtlänge \\
\hline Gruppe- $\Lambda$ & $0.161 \mathrm{~g}$ & $0.81 \mathrm{~cm}$ & $2.38 \mathrm{~cm}$ \\
Gruppe-B & 0.085 & 0.70 & 2.01 \\
Gruppe-C & 0.115 & 0.70 & 2.29 \\
Gruppe-D & 0.101 & 0.68 & 2.06 \\
\hline
\end{tabular}

Aus diesen Befunden kann man wohl schliessen, dass die Kälte $\left(10^{\circ} \mathrm{C}\right)$ den Kaulquappen von Bufo vulgaris japonicus ungünstig ist, somit die Metamorphose wesentlich verspätet wird, und auf das Wachstum hemmend einwirkt.

b. Versuch an Rana japonica und Hynobius fuscus.

Es handelt sich um die Larven von Hynobius fuscus und Kaulquappen von Rana japonica. Die Eier von beiden Tieren wurden in unserem Aquarium ausgebrütet und weiter dort aufgezogen. Die Körperlänge der Tiere beträgt durchschnittlich bei Hynobius $16 \mathrm{~mm}$, bei Kaulquappen $18 \mathrm{~mm}$. Als Nahrung wurde dem Hynobius ausschliesslich Limnodrilus gotoi gegeben. Die beiden Versuchstiere wurden in 3 Gruppen eingeteilt. In jede Gruppe kamen 20 Tiere. Die erste Gruppe wurde in einem dunklen Kühlkasten, die zweite in einem hellen Kühlkasten mit Glasfenster und die dritte als Kontroll bei Zimmertemperatur auf Arbeitstisch gefüttert. Das Experiment dauerte vom 21. April bis zum 11. Juni 1928, nämlich 80 Tage lang. Das Körpergewicht der lebenden Tiere wurde alle 7 Tage eimmal gewogen (Tabelle 4).

Tabelle 4 .

\begin{tabular}{|c|c|c|c|c|c|c|}
\hline \multirow{2}{*}{ Datum } & \multicolumn{3}{|c|}{ Hynobius fuscus } & \multicolumn{3}{c|}{ Rana japonica } \\
\cline { 2 - 5 } & $\begin{array}{c}\text { Kälte-Dunkel- } \\
\text { Tier }\end{array}$ & $\begin{array}{c}\text { Kälte- } \\
\text { Tier }\end{array}$ & $\begin{array}{c}\text { Kontroll- } \\
\text { Tier }\end{array}$ & $\begin{array}{c}\text { Kälte-Dunkel- } \\
\text { Tier }\end{array}$ & $\begin{array}{c}\text { Kailte- } \\
\text { Tier }\end{array}$ & $\begin{array}{c}\text { Kontroll- } \\
\text { Tier }\end{array}$ \\
\hline $21 / \mathrm{IV}$ & $0149 \mathrm{~g}$ & 0.139 & 0.139 & 0.036 & 0.035 & 0.034 \\
$28 / \mathrm{IV}$ & 0.166 & 0.148 & 0.196 & 0.032 & 0.032 & 0.040 \\
$5 / \mathrm{V}$ & 0.226 & 0.186 & 0.391 & 0.039 & 0.037 & 0.052 \\
\hline
\end{tabular}




\begin{tabular}{|c|c|c|c|c|c|c|}
\hline \multirow{2}{*}{ Datum } & \multicolumn{3}{|c|}{ Hynobius fuscus } & \multicolumn{3}{|c|}{ Rana japonica } \\
\hline & $\begin{array}{c}\text { Kiilte-Inunkel- } \\
\text { Tier }\end{array}$ & $\begin{array}{l}\text { Kälte- } \\
\text { Tier }\end{array}$ & $\begin{array}{l}\text { Kontroll- } \\
\text { Tier }\end{array}$ & $\begin{array}{c}\text { Kälte-Dunkel- } \\
\text { Tier }\end{array}$ & $\begin{array}{l}\text { Kälte- } \\
\text { Ties }\end{array}$ & $\begin{array}{c}\text { Kontroll- } \\
\text { Tier }\end{array}$ \\
\hline $1 \ddot{2} / \mathrm{V}$ & 11.2 .21 & 0.216 & $(0.5 .51$ & $0.04 \%$ & 0.043 & 0.053 \\
\hline $1 ! / / 2$ & 1).2!?! & $11.2+7$ & $(1.755$ & 0.041 & $(1 . ! .5]$ & 0.081 \\
\hline $26 ; / \mathrm{V}$ & $0 .: 3: 37$ & $(1.27 .5$ & $0.8+7$ & $0.0+4$ & $0.11 \%: 3$ & $0.11(10)$ \\
\hline$\because / \mathrm{VI}$ & 11.354 & 1).:31:3 & $1.114 \pi$ & 0.049 & 0.065 & 0.156 \\
\hline !I I & $(1 .: 39)$ & $11 .: 31$ & 1.4112 & 0.055 & $0.1) 71$ & $0.23: 3$ \\
\hline $16 / \mathrm{VI}$ & 0.3994 & $0.3 \times 2$ & $1.34: ?$ & - & 0.076 & $0.2(11)$ \\
\hline$\because: \because / V I$ & $(1.471)$ & $0.4: 35$ & $1.4 \backslash 1$ & - & - & (1).2.2011 \\
\hline
\end{tabular}

Beim Hynobius trat die crste Metamorphose des Kontrolltieres am 54. Vorsuchstage cin und die letzte wurde am 80. Versuchstage beendet. Die Kaulquappen der ariten und zweiten Gruppe sind bis zum 23. Juni alle zugrunde gegangen. Die Zunahme des Körperenewichts von Hynobius ist bei (rster Gruppe (Kälte-Dunkel-Tier) vi(l grösser als bei den der zweiten (Kälte-Tier); bœi Kaulquappen verhält die Sache sich ganz umgekehrt.

c. Langmitirrer Fütterungsversuch an Larven von Hynobius fuscus bei Kälte.

1)

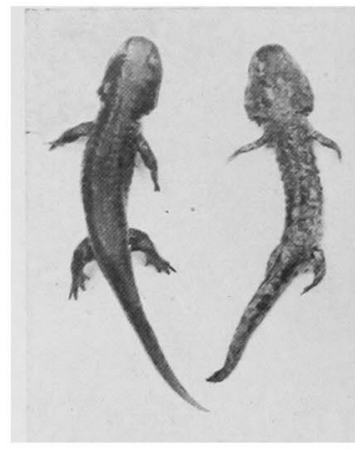

Fị. 1 .

Hynobius fuscus, $\frac{7}{10} \mathrm{mal}$ verkleinert photographiert. a. Kontrolltier im Juli 1:-7, b. neotenisches Tier hei Kälte (rom Iai $19-27$ his zum Juni 1!2!) c. 1. d neotenische Tiere bei Kälte (vom Mai 1!-7 his zum Juli 1928).
$\operatorname{Lm} 2$ 26. Mai $192 \overline{7}$ wurden 30 Larven ron Hymobius fuscus in einem Kühlkasten $\left(5^{\circ}-\right.$ $10^{\circ}$ (') untergebracht und (lort für zwei Jahre lang sorgfältig gefüttert. Die Körperläng der Larren war beim Beginn des Experimentes $29-37$ nm. Im ersten Jahre starben 17 - Tiere und 9 Tiere wandelten sich in normaler Wrise um; die Umwandlungszeit war sehr verlängert, die übrigen 
4 Tiere haben überwintert. Im zweiten Jahre starben aber 3 überwinterte Tiere, ein Ticr hat wicder überwintert, starb aber im Juli 1929 nicht motamorphosiert. Die überwinterten Tiere zeigen alle deutliche Kiemen, wie in Figur $1 \mathrm{zu}$ ersehen ist. Darans kann man schliessen, dass durch Einwirkung von Kälte $\left(5^{\circ}-10^{\circ} \mathrm{C}\right)$ beim Hynobius die unvollkommene Neotenic erfolgt. Bei Kolloidfärbung zeigte clie Schildclrüse dieser Larven stärkere Stagnation der Follikel.

B. Über die Wirkung des Schilddrüsen-Präparates auf Larven von Hynobius und Kaulquappen bei Kïlte.

Als Versuchstiore brauchte ich die Kaulquappen von Rama japonica und Bufo vulgaris sowic die Larven von Hynobius fuscus. Ich verfolgte im Jahre 1927 den Bufo und im 1928 die Rana und den Hynobius. Die Versuchstiere wurden in folgencle 4 Gruppen geteilt:

a

b)

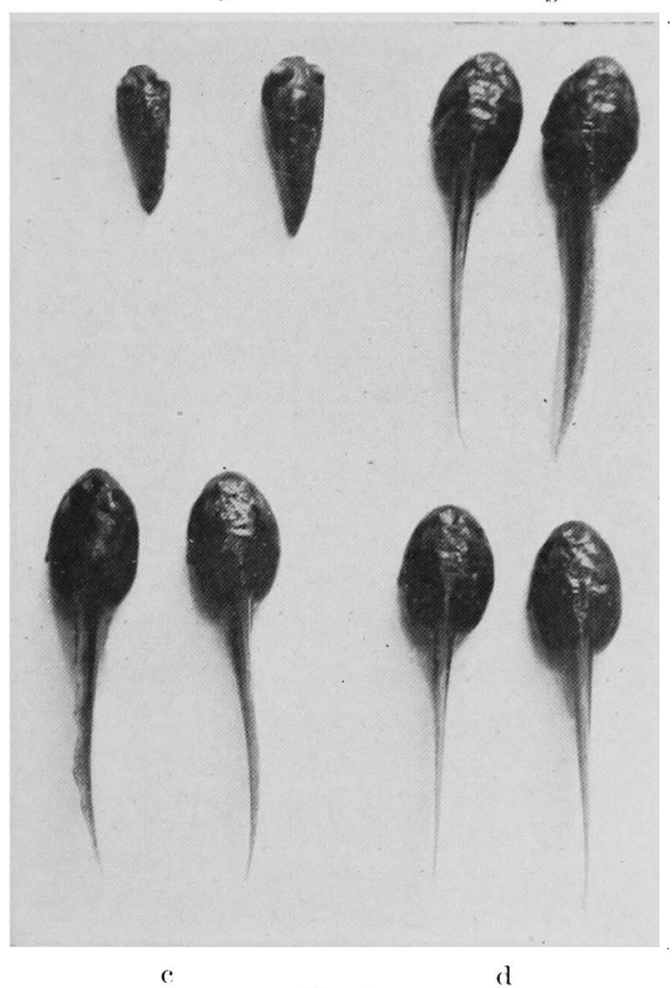

Fig. 2.

Rana japonica, auf $1 \frac{7}{10} \mathrm{mal}$ vergrössert photographiert. a. Thyreoideumtiere bei Zimmertemperatur, b. Kontrolltiere bei Zimmertemperatur, c. Thyreoideumtiere bei Kälte, d. Kontrolltiere bei Kälte. 
1. Gruppe a : Thyreoideum siccum von Sankyo Co. Tokyo und Leberpulver des Rindes, bei Zimmertemperatur gehalten,

2. (xruppe b): gleiche Menge von Thyreoideum und Leberpulver des Rindes, im Kühlkasten von $0^{\circ}-5^{\circ} \mathrm{C}$ gehalten,

3) Gruppe c: Leberpulver des Rincles, bei Zimmertemperatur gehalten,

4. Gruppe d: Leherpulver des Rindes, in Kühlkasten von $0^{\circ}-5^{\circ} \mathrm{C}$ gehalten.

a

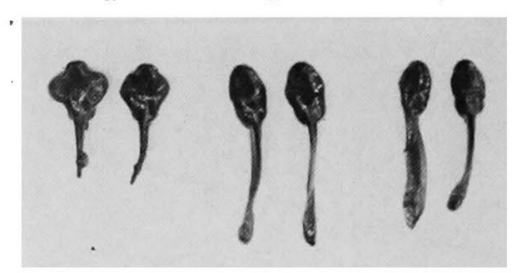

Fig. ::.

Bufo vulgaris japonicus, auf $\frac{8}{10}$ mal verkleinert photographiert. a. Thyreoideumliere bei Zimmertemperatur, b. Thyreoideumtiere bei Kälte (hell), c. Thyreoideumtiere bi kälte (dunkel).
Die Tiere von Gruppe-a zeigten schon am 3. Tersuchstage eine starke Schildrlüsenreaktion (Beschleunisrung der Differenzierung und der Metanorphose). Äuss rlich zeigt sich k.in makroskopisch bemerkbarer Interschied zwischen Ciruppe-b und d. Die genamere Unter-uchung muss natürlich mittels der mikroskopischen Forschung vervollständigt werden, worauf ich in der weiteren Mitteilung eingehen werde. Aus diesen Befunden kam man leicht zu dem Schluss kommen, dass dir Wirkung von Thyreoideum siccum durch die Kälte $\left(0^{\circ}-5^{\circ}\left(^{\prime}\right)\right.$ sehr stark behindert wirl.

\section{Schluss.}

Durch die Fütterung nit Schilddrüıs oder ihren Präparaten an Amphibienlarven wurde die Beschleunigung dor Metamorphose herbeiceführt, wie bisher bekannt. Die Wirkung bei Kaulquappen (Anuren) war viel stärker als bei Hynobiuslarven (Urodelen).

Aus diesen Resultat lässt es sich anscheinend erklären, dass die neotenischen Larv'n bei Urodelen vicl häufiger heobachtet werden als bei Anuren.

Die Wirkungslosigkeit des Thymus-Präparates auf Metamorphose spricht wahrscheinlich dafür, dass das Thymus für die Entstehung der Neotenie keine grosse Rolle spielt.

Anderen Autoren mich anschliessend möchte ich bchaupten, dass die niedrige Tomperatur für die Neotenie der Amphibienlarven nötig ist. Es ist mir nicht gelungen, dic neotenischen Larven dauernd als solche 
zu erhalten. Vielleicht war die Temperatur bei mir nicht niedrig genug gewesen. Dass die Neotenie, Schilddrüsenfunktion und die niedrige Temperatur miteinander in enger Beziehung stehen, ist aus meinem Versuch III sicher zu ersehen. Daraus erhellt, dass die Schilddrüsenfunktion durch die niedrige Temperatur stark beeinflusst wird, wodurch die Verspätung der Metamorphose oder Neotenie herbeigeführt wird.

Zum Schluss spreche ich Herrn Professor K. Okajima für seine stete liebenswürdige Unterstützung bei dieser Arbeit meinen herzlichsten Dank aus.

\section{Literatur.}

1. Adler, I.., Untersuchungen über die Entstehung der Anphibienneotenie. Pflüger's Jrch. Bd. 39. 1916.

$\because$ —— S(Childdrüse und Wärmeregulation (Untersuchungen an Winterschläfern). Arch. f. Exper. Pathol. u. Pharmakol. Bd. 85. 1920.

3. Bocttger, C. R. und Schwarz, E., Über neotenische Larven des Teichmolchs. Zool. In\%. Bd. 78. 1928.

4. von Chauvin, M., Über die Verwandlung der mexikanischen Axolotl in Amblystoma. Zeitschr. f. wiss. Zool. Til. $27 . \quad 1876$.

5. Cramer, W., On the thyroid-adrenal apparatus and its function in the heat regulation of the body. Journ. of Physiol. Vol. 50. 1915-16.

6. Duméril, 1., Méta. les Batr. ourodèles à branch. extér, du Méxique dits Axolotls. Ann. Sci. Nat. Zool. 7. 1867. (zitiert bei Sasaki).

7. *Fremery, P. de, Over neotenie bij Triton taeniatus Laur. Acad. Proefschr. 1928. Bussum.

S. Gadlow, H., Imphibia and Reptiles. Cambridge Nat. Hist. 8. 1909.

9. Gudernatsch, J. E., Studies on Internal secretion. IV. Treatment of Tadpoles with Thyroid and Thymus Extracts. Inat. Rec. Vol. 11. 1916.

10. H amann, O., Über kiementragende Tritonen. Jenaische Zeitschr. f. Naturwiss. Bd. 1t. 1880. (zitiert bei Sasaki).

11. Hart, C., Beiträge zur biologischen Bedeutung der innersekretorischen Organe. ․ Mitteilungen. Der Einfluss abnormer Aussentemperaturen auf Schilddrüse und Hoden. Pflüger's Arch. Bł1. 196. 1922.

12. *Huxley, .T. S., Thyroid and temperature in cold-blooded Vertebrates. Nature London. Vol. 129. 1929).

13. Ingram, W. R., Studies on amphibian neoteny, Journ. Exper. Zool. Vol. 53. 1929 .

14. *__, Studies on amphibian neoteny. Physiol. Zool. 1929.

15. Kammerer, P., Beitrag zur Erkenntnis der Verwandtschaftverhältnisse von Salamandra atra und maculosa. Irch. f. Entw.-mech. Bd. 17. 1904.

16. Mills, Am. Journ. Physiol. Vol. 45. 1918. (zitiert bei Nishimura).

* Diese Arbciten waren mir nur als Referate zugänglich. 
17. Nishimura, S., Nitta, K. und Minouchi, T., L̇her den Finfluss der Auscentemperatur auf die innersekretorischen (rgane. Folia Endocrinol. Japonica. Bis. 4. 1928

18. Sasaki, M., On a Japanese salamander, in lake Kuttarush, which propagate like the Axolotl. Journ. of the Coll. of Agr. Hokkaido Imp. Univer. Vol. 15. $19 \div 4$.

19. Swingle, W. W., Fxperiments on the metamorphosis of the neotenous amphibians Journ. Exper. Zool. Vol, 3t. 1920.

20. —, The acceleration of metamorphosis in frog larvae by thyroid feeding and the effects upon the alimentary tract and sex gland. Journ. Exper. Zool. Vol. 24. 1917-1s.

21. Uhlenhuth, E., Regeneration and Neoteny. Journ. Gen. Physiol. Vol. 2. 1920.

2.). Weismann, 1., Üher die Umwandlung des mexikanischen Axolotl in ein Amblystoma. Zeitschr. f. wiss. Zool. Bd. 25. 1s.5.

29. Woltersdorff, W., Über die Nestenie der Batrachier. Zool. (iart. Bd. :3. 1si6. (zitiert bei sasaki).

24. Zeller, E., Zur Vertenie der Tritonen. Jahresl. Ver. f. vaterl. Naturk. Wurttemberg. 1s99. (zitiert bei sasaki). 